\title{
Системы натурального вывода для некоторых логик с истинностными провалами и логик с пресыщенными оценками ${ }^{1}$
}

\author{
В. О. ШАНГИН
}

\begin{abstract}
In the paper we present Fitch-style natural deduction systems of some logics with truth-value gluts and truth-value gaps. We show that natural deduction systems of the logics in question can be set up with different formulations of either the disjunction elimination rule or the negation introduction rule. We give a constructive proof that for each natural deduction system $\mathrm{N}$ of a logic $\alpha$, a formula $\mathrm{A}$ is $\mathrm{N}$-provable iff $\mathrm{A}$ is a $\alpha$-theorem.
\end{abstract}

Ключевые слова: теория доказательств, натуральный вывод, неклассическая логика, логики с истинностными провалами и с пресыщенными оценками, правило исключения дизъюнкции

\section{Введение}

Задается стандартный пропозициональный язык $L$ с алфавитом $\left\{p_{1}, q_{1}, r_{1}, p_{2}, \ldots,(),, \wedge, \vee, \supset, \neg\right\}$. Определение формулы языка $L$ обычно. Буквы $A, B, C, D, A_{1}, \ldots$ обозначают произвольные формулы языка $L$. Буквы $\Gamma, \Delta, \Gamma_{1}, \ldots$ обозначают произвольные конечные множества формул языка $L$.

В работе используются аксиоматические системы для некоторых логик с истинностными провалами и логик с пресыщенными оценками. Мы используем аксиоматическую систему HPar для логики Par [3], аксиоматическую систему HPCont для логики PCont [5], аксиоматическую систему НPComp для логики

\footnotetext{
${ }^{1}$ Исследование выполнено при финансовой поддержке РГНФ в рамках научно-исследовательского проекта РГНФ («Логики с истинностными провалами и логики с пресыщенными оценками: семантики обобщенных оценок и аксиоматизации посредством исчислений с правилами оперирования комплексами логических связок»), проект № 10-03-00570а.
} 
PComp [1] и аксиоматическую систему HPContPComp для логики PContPComp [4]. Объем статьи не позволяет дать описание данных работ, поэтому их знание предполагается. Некоторые результаты данной работы представлены без доказательств в [6].

Статья структурирована следующим образом. В параграфе 1 задается формулировка системы натурального вывода NPar для логики Par. На основе NPar с использованием различных ограничений для правила введения отрицания задаются формулировки систем натурального вывода NPCont, NPComp и NPContPComp для логик PCont, PComp и PContPComp соответственно. Параграф 2 посвящен метатеоретическим свойствам систем, предложенным в параграфе 1 . В параграфе 3 на основе NPar с использованием различных ограничений для правила исключения дизъюнкции задаются формулировки систем натурального вывода NPCont*, NPComp* и NPContPComp* для логик PCont, PComp и PContPComp соответственно. Параграф 4 посвящен метатеоретическим свойствам систем, предложенным в параграфе 3.

\section{1 Система натурального вывода для логики Par и системы натурального вывода с правилом введения отрицания для логик PCont, PComp и PContPComp}

Cистема натурального вывода NPar. Задаются следующие NPar-правила (формула слева от «/» называется посылкой, а формула справа от «/»- заключением $)^{2}$. Отметим, что, напри-

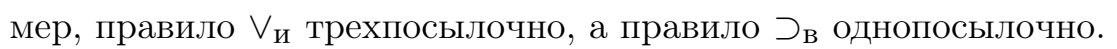
$\wedge_{\text {и } 1}: A \wedge B / A ; \wedge_{\text {и } 2}: A \wedge B / B ; \wedge_{\mathrm{B}}: A, B / A \wedge B$

$\vee_{\mathrm{n}}: A \vee B,[A] C,[B] C / C ; \vee_{\mathrm{B} 1}: A / A \vee B ; \vee_{\mathrm{B} 2}: B / A \vee B$;

$\supset_{\text {и }}: A \supset B, A / B ; \supset_{\mathrm{B}}:[C] B / C \supset B ; \supset_{\mathrm{p}}:[A \supset B] A / A^{3}$;

$\neg \neg_{\mathrm{n}}: \neg \neg A / A ; \neg \neg_{\mathrm{B}}: A / \neg \neg A ; \neg \vee_{\text {и1 }}: \neg(A \vee B) / \neg A$;

$\neg \vee_{\text {и } 2}: \neg(A \vee B) / \neg B ; \neg \vee_{\mathrm{B}}: \neg A, \neg B / \neg(A \vee B)$;

$\neg \wedge_{\mathrm{n}}: \neg(A \wedge B) / \neg A \vee \neg B ; \neg \wedge_{\mathrm{B} 1}: \neg A / \neg(A \wedge B) ; \neg \wedge_{\mathrm{B} 2}: \neg B /$

$\neg(A \wedge B) ; \neg \supset_{\mathrm{n}}: \neg(A \supset B) / A \wedge \neg B ; \neg \supset_{\mathrm{B}}: A \wedge \neg B / \neg(A \supset B)$.

\footnotetext{
${ }^{2}$ Данные правила делятся на правила введения и исключения логической связки или комплекса логических связок.

${ }^{3}$ Правило Пирса, Јp , ошибочно не добавлено ко всем системам натурального вывода в [6], [7].
} 
ОПРЕДЕЛЕНИЕ 1. ВЫводом в системе NPar (NPar-выводом) называется непустая конечная последовательность формул, удовлетворяющая следующим условиям: каждая формула есть либо посылка, либо получена из предыдущих по одному из NParправил; при применении правила $\supset_{\text {в }}(\supset$ p $)$ все формулы, начиная с последней неисключенной посылки $C(A \supset B)$ и вплоть до результата применения данного правила, исключаются из дальнейших шагов вывода; при применении правила $\vee_{\text {и }}$ все формулы, начиная с посылки $A$ и вплоть до формулы $C$, а также все формулы, начиная с посылки $B$ и вплоть до формулы $C$, исключаются из дальнейших шагов вывода.

NPar-выводом формулы $B$ из непустого множества посылок $\Gamma$ (сокращенно $\Gamma \vdash_{N \text { Par }} B$ ) называется NPar-вывод, в котором множество неисключенных посылок есть Г и последняя формула есть формула $B$. NPar-доказательством формулы $B$ называется NPar-вывод формулы $B$ из пустого множества неисключенных посылок. NPar-теоремой называется формула $B$, для которой имеется NPar-доказательство.

Рассмотрим двухпосылочное правило введения отрицания $\neg_{\mathrm{B}}$ : $[C] D, \neg D / \neg C$. Добавление правила $\neg_{\mathrm{B}} \mathrm{K}$ множеству NParправил вместе с требованием, что формула $C$ - это последняя неисключенная посылка в выводе, дает систему натурального вывода для классической логики высказываний адекватную системе NP, предложенной в [2]. C другой стороны, мы рассмотрим три правила введения отрицания (PCont $\neg_{\mathrm{B}}$, PComp $\neg_{\mathrm{B}}$ и PContPComp $\neg_{\text {в }}$ ), которые получаются введением различных ограничений на вид формулы С в правиле $\neg_{\mathrm{B}}$. Мы покажем, что добавление правила PCont $\neg_{\mathrm{B}}\left(\mathrm{PComp} \neg_{\mathrm{B}} / \mathrm{PContPComp} \neg_{\mathrm{B}}\right)$ к множеству NPar-правил вместе с требованием, что формула $C$ - это последняя неисключенная посылка в выводе, дает систему натурального вывода NPCont (NPComp / NPContPComp) для неклассической пропозициональной логики PCont (PComp / PContPComp). Данный подход аналогичен [3], где различные ограничения на вид основной секвенции дают секвенциальные исчисления $\mathrm{GK}_{2}$, GPar, GPCont для классической логики высказываний и неклассических пропозициональных логик Par и PCont.

Система натурального вывода NPCont (NPComp) NPContPComp). Добавляем к множеству NPar-правил правило 
$\mathbf{P C o n t} \neg_{\mathbf{B}}\left(\mathbf{P C o m p} \neg_{\mathbf{B}} / \mathbf{P C o n t P C o m p} \neg_{\mathbf{B}}\right):[C] D, \neg D / \neg C$, где $C$ имеет вид $A \vee \neg A(\neg A \supset(A \supset B) /(A \wedge \neg A) \supset(B \vee \neg B))$.

ОПРЕДЕЛЕНИЕ 2. ВЫводом в системе NPCont (NPComp / NPContPComp) называется непустая конечная последовательность формул, удовлетворяющая условиям определения 1 , а также следующему условию: при применении PCont $\neg_{\mathrm{B}}\left(\mathrm{PComp} \neg_{\mathrm{B}}\right.$ / PContPComp $\neg_{\text {в }}$ ) все формулы, начиная с последней неисключенной посылки С и вплоть до результата применения данного правила, исключаются из дальнейших шагов вывода.

NPCont (NPComp / NPContPComp)-выводом формулы $B$ из непустого множества посылок $\Gamma$ (сокращенно $\left.\Gamma \quad \vdash_{\text {NPCont }(N P C o m p / N P C o n t P C o m p)} B\right)$ называется NPCont (NPComp / NPContPComp)-вывод, в котором множество неисключенных посылок есть $Г$ и последняя формула есть формула $B$. NPCont (NPComp / NPContPComp)-доказательством формулы $B$ называется NPCont (NPComp / NPContPComp)-вывод формулы $B$ из пустого множества неисключенных посылок. NPCont (NPComp / NPContPComp)-теоремой называется формула $B$, для которой имеется NPCont (NPComp/NPContPComp)доказательство.

\section{2 Метатеоретические свойства NPar, NPCont, NPComp и NPContPComp}

Для исследования метатеоретических свойств NPar, NPCont, NPComp и NPContPComp мы используем аксиоматические системы HPar, HPCont, HPComp и HPContPComp для логик Par, PCont, PComp и PContPComp $[3,5,1,4]$. Отметим, что в любой из данных аксиоматических систем доказуемы формулы $((A \wedge B) \supset C) \supset(A \supset(B \supset C)),(A \supset(B \supset C)) \supset((A \wedge B) \supset C)$ и имеется теорема дедукции.

TEOPEMA 1. $\Gamma \vdash_{N P a r} A \Leftrightarrow \Gamma \vdash_{H P a r} A$.

Доказательство. Доказательство $\Leftarrow$. Достаточно показать, что все HPar-правила выводимы в NPar. Рассмотрим правила $(A \supset C) \supset((B \supset C) \supset((A \vee B) \supset C))$ и $((A \supset B) \supset A) \supset A$. Остальные случаи аналогичны.

$$
\vdash_{N P a r}(A \supset C) \supset((B \supset C) \supset((A \vee B) \supset C))
$$


1. $A \supset C-$ посылка

2. $B \supset C$ - посылка

3. $A \vee B-$ посылка

4. $A-$ посылка

5. $C-\supset_{\text {и }}: 1,4$

6. $B-$ посылка

7. $C-\supset_{\text {и }}: 2,6$

8. $C-\vee_{\text {й }}: 3,5,7[4-5],[6-7]$

9. $(A \vee B) \supset C-\supset_{\mathrm{B}}: 8[3-8]$

10. $(B \supset C) \supset((A \vee B) \supset C)-\supset_{\mathrm{B}}: 9[2-9]$

11. $(A \supset C) \supset((B \supset C) \supset((A \vee B) \supset C))-\supset_{\mathrm{B}}: 10[1-10]$

$\vdash_{N P a r}((A \supset B) \supset A) \supset A$

1. $(A \supset B) \supset A-$ посылка

2. $A \supset B-$ посылка

3. $A-\supset_{\mathrm{n}}: 1,2$

4. $A-\supset_{\mathrm{p}}: 3[2-3]$

5. $((A \supset B) \supset A) \supset A-\supset_{\mathrm{B}}: 4[1-4]$

Доказательство $\Rightarrow$. Покажем сначала лемму 1 .

ЛEMMA 1. $\Gamma \vdash_{N \text { Par }} A \Rightarrow \vdash_{H \text { Par }}\left(\Gamma^{\wedge}\right) \supset A$, где $\left(\Gamma^{\wedge}\right)-$ эmo $\wedge$ образ множества формул Г, заданный следующим образом:

Если Г пусто, то $\left(\Gamma^{\wedge}\right) \supset$ A есть $A$;

Если $\Gamma=\left\{D_{1}, D_{2}, \ldots, D_{m}\right\}, m \geq 1, m o\left(\Gamma^{\wedge}\right) \supset A$ есть $\left(D_{1} \wedge\right.$ $\left.\left(D_{2} \wedge \ldots \wedge D_{m}\right) \ldots\right) \supset \mathrm{A}$. 
Доказательство. Доказательство ведется методом двойной математической индукции по длине $n$ и числу применений $s$ NParправил в $\Gamma \vdash_{N \text { Par }} A$.

Базис: $s=0$. Тогда $A \in \Gamma$.

Этому выводу соответствует HPar-доказательство формулы $\left(\Gamma^{\wedge}\right) \supset A$ :

1. $\left(\Gamma^{\wedge}\right) \supset A-$ HPar-аксиома $^{4}$

Итак, мы доказали лемму для всех NPar-выводов, в которых нет ни одного применения NPar-правил.

Теперь предположим (IH): лемма верна для всеx NPar-выводов с числом $d$ применений NPar-правил, $d \leq s$. Покажем, что лемма верна для всех NPar-выводов с числом $s+1$ применений NParправил.

Число рассматриваемых случаев зависит от числа NPar-правил ${ }^{5}$.

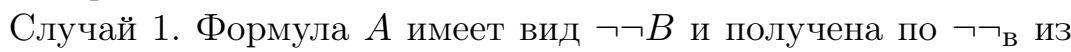
$B_{i}, 1 \leq i<n+1$.

Тогда $\Gamma \vdash_{N P a r} A$ принимает следующий вид:

$\Gamma$

$\cdots$

i. $B$

$\cdots$

$\mathrm{n}+1 . \neg \neg B-\neg \neg_{\mathrm{B}}: \mathrm{i}$

Этому выводу соответствует HPar-доказательство формулы $\left(\Gamma^{\wedge}\right) \supset \neg \neg B:$

i'. $\left(\Gamma^{\wedge}\right) \supset B-$ HPar-теорема (IH)

i'+1. $B \supset \neg \neg B-$ HPar-аксиома

\footnotetext{
${ }^{4}$ Без потери общности, данная формула является частным случаем HPar-аксиомы $(A \wedge B) \supset B$, если $n \geq 2$. Если $n=1$, то данная формула является HPar-аксиомой $A \supset A$.

${ }^{5}$ Аналогичные случаи мы пропустим.
} 
i'+2. $\left(\left(\Gamma^{\wedge}\right) \supset B\right) \supset\left((B \supset \neg \neg B) \supset\left(\left(\Gamma^{\wedge}\right) \supset \neg \neg B\right)\right)$ - HParаксиома

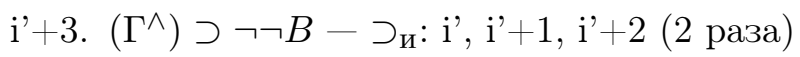

Случай 2. Формула А имеет вид $B_{k} \supset B_{j}$ и получена по $\supset_{\text {в }}$ из $B, 1 \leq j<n+1,1 \leq k<n+1$.

Тогда $\Gamma \vdash_{N P a r} A$ принимает следующий вид:

$\Gamma$

k. $C$ - посылка

$\cdots$

j. $B$

$\mathrm{n}+1 . C \supset B-\supset_{\mathrm{B}}: \mathrm{j}[\mathrm{k}-\mathrm{n}]$

Этому выводу соответствует HPar-доказательство формулы $\left(\Gamma^{\wedge}\right) \supset(\mathrm{C} \supset B):$

j'. $\left(\left(\Gamma^{\wedge}\right) \wedge C\right) \supset B-$ HPar-теорема $(\mathrm{IH})$

j'+1. $\left(\left(\left(\Gamma^{\wedge}\right) \wedge C\right) \supset B\right) \supset\left(\left(\Gamma^{\wedge}\right) \supset(C \supset B)\right)$ - HPar-теорема

$\mathrm{j}^{\prime}+2 .\left(\Gamma^{\wedge}\right) \supset(C \supset B)-\supset_{\mathrm{H}}: \mathrm{j}^{\prime}, \mathrm{j}^{\prime}+1$

Q.E.D.

Теорема 1 следует из леммы 1 и $\Gamma \vdash_{H \text { Par }} A \Leftrightarrow \vdash_{H P a r}\left(\Gamma^{\wedge}\right) \supset A$.

Q.E.D.

TEOPEMA 2. $\Gamma \vdash_{N P C o n t} A \Leftrightarrow \Gamma \vdash_{H P C o n t}\left(\Gamma^{\wedge}\right) \supset A$

Доказательство. Доказательство $\Leftarrow$. Достаточно дополнить случай $\Leftarrow$ теоремы 1 NPCont-доказательством $A \vee \neg A$.

1. $\neg(A \vee \neg A)-$ посылка 
2. $A-\neg \vee_{\text {и } 1}: 1$

3. $\neg A-\neg \vee_{\text {и2}}: 1$

4. $\neg \neg(A \vee \neg A)-\mathrm{PCont} \neg_{\mathrm{B}}: 2,3[1-3]$

5. $A \vee \neg A-\neg_{\mathrm{n}}: 4$

Доказательство $\Rightarrow$. Покажем сначала лемму 2 .

ЛEMMA 2. $\Gamma \vdash_{N \text { PCont }} A \Rightarrow \vdash_{H P C o n t}\left(\Gamma^{\wedge}\right) \supset A$.

Доказательство. K рассмотренным в лемме 1 случаям добавляется следующий.

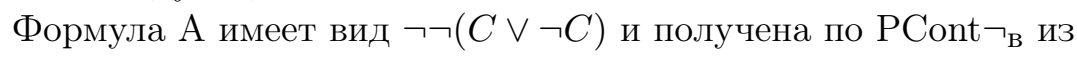
$B_{k}$ и $B_{h}, 1 \leq \mathrm{k}<\mathrm{n}+1,1 \leq \mathrm{h}<\mathrm{n}+1, \mathrm{~m}<\mathrm{n}+1$.

Тогда $\Gamma \vdash_{N P C o n t} A$ принимает следующий вид:

$\Gamma$

$\cdots$

m. $\neg(C \vee \neg C)-$ посылка

k. $B$

h. $\neg B$

$\cdots$

$\mathrm{n}+1 . \neg \neg(C \vee \neg C)-\neg_{\mathrm{B}}: \mathrm{k}, \mathrm{h}[\mathrm{m}-\mathrm{n}]$

Этому выводу соответствует HPCont-доказательство формулы $\left(\Gamma^{\wedge}\right) \supset \neg \neg(C \vee \neg C)$ :

k'. $\left(\left(\Gamma^{\wedge}\right) \supset \neg(C \vee \neg C)\right) \supset B_{k}-$ HPCont-теорема (IH)

$\mathrm{k}^{\prime}+1 . \quad\left(\left(\Gamma^{\wedge}\right) \supset \neg(C \vee \neg C)\right) \supset \neg B_{k}-$ HPCont-теopema (IH)

$\mathrm{k}^{\prime}+2 . C \vee \neg C-$ HPCont-аксиома

$\mathrm{k}^{\prime}+3 .(C \vee \neg C) \supset \neg \neg(C \vee \neg C)-$ HPCont-аксиома 
$\mathrm{k}^{\prime}+4 . \quad \neg \neg(C \vee \neg C)-\supset_{\mathrm{n}}: \mathrm{k}^{\prime}+2, \mathrm{k}^{\prime}+3$

$\mathrm{k}^{\prime}+5 . \neg \neg(C \vee \neg C) \supset\left(\left(\Gamma^{\wedge}\right) \supset \neg \neg(C \vee \neg C)\right)$ - HPCont-аксиома

$\mathrm{k}^{\prime}+6 . \quad\left(\Gamma^{\wedge}\right) \supset \neg \neg(C \vee \neg C)-\supset_{\mathrm{n}}: \mathrm{k}^{\prime}+4, \mathrm{k}^{\prime}+5$

Q.E.D.

Теорема 2 следует из леммы 2 и $\Gamma \vdash_{\text {HPCont }} A \Leftrightarrow \vdash_{\text {HPCont }}$ $\left(\Gamma^{\wedge}\right) \supset A$.

Q.E.D.

TEOPEMA 3. $\Gamma \vdash_{N P C o m p} A \Leftrightarrow \Gamma \vdash_{H P C o m p}\left(\Gamma^{\wedge}\right) \supset A$

Доказательство. Доказательство аналогично доказательству теоремы 2.

Q.E.D.

TEOPEMA 4. $\Gamma \vdash_{N P C o n t P C o m p} A \Leftrightarrow \Gamma \vdash_{H P C o n t P C o m p}\left(\Gamma^{\wedge}\right) \supset A$

Доказательство. Доказательство аналогично доказательству теоремы 2.

Q.E.D.

\section{3 Системы натурального вывода с правилом исключения дизъюнкции для логик PCont, PComp и PContPComp}

В данном параграфе мы зададим альтернативные формулировки систем натурального вывода NPCont*, NPComp* и NPContPComp* для логик PCont, PComp и PContPComp. В отличие от формулировок систем натурального вывода из параграфа 2, где главную роль играет правило введения отрицания, в данных формулировках главную роль играет правило исключения дизъюнкции.

Рассмотрим следующие двухпосылочные правила исключения дизгюнкиии: PCont $\vee_{\mathbf{n}}:[A] C,[\neg A] C / C ; \mathbf{P C o m p} \vee_{\mathbf{n}}: A \vee$ $B, \neg A / B$ и PContPComp $\vee_{\mathbf{и}}: A \vee(B \vee \neg B), \neg A / B \vee \neg B$. Покажем, что добавление правила $\mathrm{PCont}_{\text {и }}\left(\mathrm{PComp}_{\text {и }} /\right.$ PContPComp $\vee_{\text {и }}$ ) к множеству NPar-правил дает систему натурального вывода NPCont* (NPComp* / NPContPComp*) для логики PCont (PComp / PContPComp). 
Система натурального вывода NPCont* (NPComp* / NPContPComp*). Добавляем к множеству NPar-правил правило $\mathrm{PCont}_{\text {и }}\left(\mathrm{PComp}_{\text {и }} / \mathrm{PCont} \mathrm{PComp} \vee_{\text {и }}\right)$.

ОПРЕДЕЛЕНИЕ 3. ВЫводом в системе NPComp* (NPContРComp*) называется непустая конечная последовательность формул, удовлетворяющая условиям определения 1. Выводом в системе NPCont* называется непустая конечная последовательность формул, удовлетворяющая условиям определения 1, а также следующему условию: при применении правила $\mathrm{PCont} \mathrm{V}_{\text {и }}$ и все формулы, начиная с посылки $A$ и вплоть до формулы $C$, а также все формулы, начиная с посылки $\neg A$ и вплоть до формулы $C$, исключаются из дальнейших шагов вывода.

NPCont* (NPComp* / NPContPComp*)-выводом формулы B из непустого множества посылок $\Gamma$ (сокращенно $\left.\Gamma \vdash_{\text {NPCont } * \text { (NPComp*/NPContPCont } *)} \quad B\right)$ называется NPCont* (NPComp* / NPContPComp*)-вывод, в котором множество неисключенных посылок есть Г и последняя формула есть формула B. NPCont* (NPComp* / NPContPComp*)-доказательством формулы B называется NPCont* (NPComp* / NPContPComp*)вывод формулы В из пустого множества неисключенных посылок. NPCont* (NPComp* / NPContPComp*)-теоремой называется формула В, для которой имеется NPCont* (NPComp* / NPContPComp*)-доказательство.

\section{4 Метатеоретические свойства NPCont*, NPComp* и NPContPComp*}

Аналогично параграфу 2, для исследования метатеоретических свойств NPCont*, NPComp* и NPContPComp* используем аксиоматические системы HPCont, HPComp и HPContPComp [3, $5,1,4]$.

TEOPEMA 5. $\Gamma \vdash_{N P C o n t *} A \Leftrightarrow \Gamma \vdash_{H P C o n t}$.

Доказательство. Доказательство $\Leftarrow$. Достаточно дополнить случай $\Leftarrow$ теоремы 1 NPCont*-доказательством $A \vee \neg A$.

1. $A-$ посылка

2. $A \vee \neg A-\vee_{\mathrm{B} 1}: 1$ 
3. $\neg A-$ посылка

4. $A \vee \neg A-\vee_{\mathrm{B} 2}: 3$

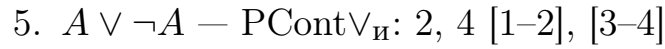

Доказательство $\Rightarrow$. Покажем сначала лемму 3.

JEMMA 3. $\Gamma \vdash_{N P C o n t *} A \Rightarrow \vdash_{H P C o n t}\left(\Gamma^{\wedge}\right) \supset A$.

Доказательство. K рассмотренным в лемме 1 случаям добавляется следующий: формула $A$ имеет вид $C$ и получена по PCont $_{\text {и из }} B_{k}$ и $B_{h}, m<n+1, s<n+1,1 \leq k<n+1$, $1 \leq h<n+1$.

Тогда $\Gamma \vdash_{N P C o n t *} A$ принимает следующий вид:

$\Gamma$

m. $B-$ посылка

k. $C$

$\cdots$

s. $\neg B-$ посылка

h. $C$

$\mathrm{n}+1 . C-\mathrm{PCont}_{\text {й }}: \mathrm{k}, \mathrm{h}[\mathrm{m}-\mathrm{k}],[\mathrm{s}-\mathrm{h}]$

Этому выводу соответствует HPCont-доказательство формулы $\left(\Gamma^{\wedge}\right) \supset C$ :

k'. $\left(\left(\Gamma^{\wedge}\right) \wedge B\right) \supset C-$ HPCont-теорема $(\mathrm{IH})$

$\mathrm{k}^{\prime}+1 .\left(\left(\Gamma^{\wedge}\right) \wedge \neg B\right) \supset C-$ HPCont-теорема (IH) 


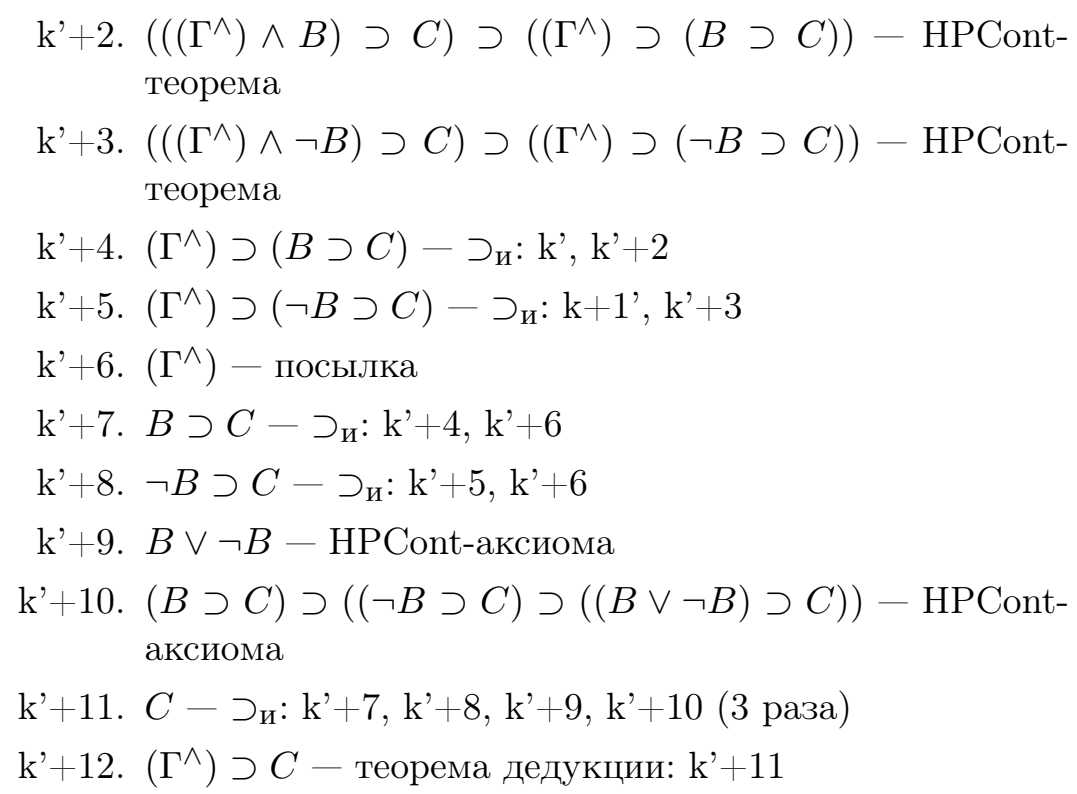

Q.E.D.

Теорема 5 следует из леммы 3 и $\Gamma \vdash_{\text {HPCont }} A \Leftrightarrow \vdash_{\text {HPCont }}$ $\left(\Gamma^{\wedge}\right) \supset A$.

Q.E.D.

TEOPEMA 6. $\Gamma \vdash_{N P C o m p *} A \Leftrightarrow \Gamma \vdash_{H P C o m p} A$.

Доказательство. Доказательство $\Leftarrow$. Достаточно дополнить случай $\Leftarrow$ теоремы 1 NPComp*-доказательством $\neg A \supset(A \supset B)$.

1. $\neg A-$ посылка

2. $A$ - посылка

3. $A \vee B-\vee_{\mathrm{B} 1}: 2$

4. $B-\mathrm{PComp \vee}_{\text {и }}: 1,3$

5. $A \supset B-\supset_{\mathrm{B}}: 4[2-4]$

6. $\neg A \supset(A \supset B)-\supset_{\mathrm{B}}: 5[1-5]$

Доказательство $\Rightarrow$. Покажем сначала лемму 4 .

ЛEMMA 4. $\Gamma \vdash_{N P C o m p *} A \Rightarrow \vdash_{H P C o m p}\left(\Gamma^{\wedge}\right) \supset A$. 
Доказательство. $\mathrm{K}$ рассмотренным в лемме 1 случаям добавляется следующий: формула $A$ имеет вид $C$ и получена по $\mathrm{PComp}_{\text {и из }} B_{k}$ и $B_{h}, 1 \leq k<n+1,1 \leq h<n+1$.

Тогда $\Gamma \vdash_{N P C o m p *} A$ принимает следующий вид:

$\Gamma$

$\cdots$

k. $A \vee C$

h. $\neg A$

n+1. $C-\mathrm{PComp} \vee$ и $_{\mathrm{k}} \mathrm{k}, \mathrm{h}$

Этому выводу соответствует НРСотр-доказательство формулы $\left(\Gamma^{\wedge}\right) \supset C$ :

k'. $\left(\Gamma^{\wedge}\right) \supset A \vee C-$ HРComp-теорема (IH)

$\mathrm{k}^{\prime}+1 .\left(\Gamma^{\wedge}\right) \supset \neg A-$ HРComp-теорема (IH)

$\mathrm{k}^{\prime}+2 . \neg A \supset(A \supset C)-$ HРComp-аксиома

$\mathrm{k}^{\prime}+3 .\left(\left(\Gamma^{\wedge}\right) \supset \neg A\right) \supset\left((\neg A \supset(A \supset C)) \supset\left(\left(\Gamma^{\wedge}\right) \supset(A \supset\right.\right.$ $C)))$ - НРСотр-аксиома

$\mathrm{k}^{\prime}+4 .\left(\Gamma^{\wedge}\right) \supset(A \supset C)-\supset_{\mathrm{n}}: \mathrm{k}^{\prime}+1, \mathrm{k}^{\prime}+2, \mathrm{k}^{\prime}+3(2$ раза $)$

$\mathrm{k}^{\prime}+5$. $C \supset C-$ НРСотр-аксиома

$\mathrm{k}^{\prime}+6 .(A \supset C) \supset((C \supset C) \supset((A \vee C) \supset C))-$ HPCompаксиома

$\mathrm{k}^{\prime}+7 .\left(\Gamma^{\wedge}\right)-$ посылка

$\mathrm{k}^{\prime}+8 . A \vee C-\supset_{\text {и }}: \mathrm{k}^{\prime}, \mathrm{k}^{\prime}+7$

$\mathrm{k}^{\prime}+9 . \quad A \supset C-\supset_{\mathrm{n}}: \mathrm{k}^{\prime}+4, \mathrm{k}^{\prime}+7$

$\mathrm{k}^{\prime}+10 . C-\supset_{\mathrm{n}}: \mathrm{k}^{\prime}+5, \mathrm{k}^{\prime}+6, \mathrm{k}^{\prime}+8, \mathrm{k}^{\prime}+9$ (3 раза)

$\mathrm{k}^{\prime}+11 .\left(\Gamma^{\wedge}\right) \supset C-$ теорема дедукции: $\mathrm{k}^{\prime}+10$

Q.E.D. 


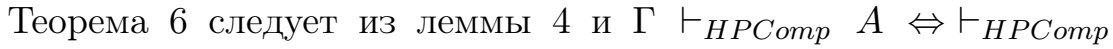
$\left(\Gamma^{\wedge}\right) \supset A$.

TEOPEMA 7. $\Gamma \vdash_{N P C o n t P C o m p *} A \Leftrightarrow \Gamma \vdash_{H P C o n t P C o m p} A$.

Доказательство. Доказательство $\Leftarrow$. Достаточно дополнить случай $\Leftarrow$ теоремы 1 NPContPComp*-доказательством $(A \wedge \neg A) \supset$ $(B \vee \neg B)$.

1. $A \wedge \neg A-$ посылка

2. $A-\wedge_{\text {и1 }}: 1$

3. $\neg A-\wedge_{\text {и } 2: 1}$

4. $A \vee(B \vee \neg B)-\vee_{\mathrm{B} 1}: 2$

5. $B \vee \neg B-\mathrm{PContPComp}_{\text {й }}: 3,4$

6. $(A \wedge \neg A) \supset(B \vee \neg B)-\supset_{\mathrm{B}}: 5[1-5]$

Доказательство $\Rightarrow$. Покажем сначала лемму 5 .

ЛEMMA 5. $\Gamma \vdash_{N P C o n t P C o m p *} A \Rightarrow \vdash_{H P C o n t P C o m p}\left(\Gamma^{\wedge}\right) \supset A$.

Доказательство. $\mathrm{K}$ рассмотренным в лемме 1 случаям добавляется следующий: формула А имеет вид $C \vee \neg C$ и получена по PContPComp $\vee_{\text {и из }} B_{k}$ и $B_{h}, 1 \leq k<n+1,1 \leq h<n+1$.

Тогда $\Gamma \vdash_{N P C o n t P C o m p *} A$ принимает следующий вид:

$\Gamma$

k. $A \vee(C \vee \neg C)$

h. $\neg A$

$\mathrm{n}+1 . C \vee \neg C-\mathrm{PContPComp}_{\text {и }}: \mathrm{k}, \mathrm{h}$ 
Этому выводу соответствует HPContPComp-вывод формулы $\left(\Gamma^{\wedge}\right) \supset(C \vee \neg C)$ :

$$
\begin{aligned}
& \text { k'. }\left(\Gamma^{\wedge}\right) \supset(A \vee(C \vee \neg C))-\text { HPContPComp-теopema (IH) } \\
& \mathrm{k}^{\prime}+1 .\left(\Gamma^{\wedge}\right) \supset \neg A-\text { HPContPComp-теорема (IH) } \\
& \mathrm{k}^{\prime}+2 .\left(\Gamma^{\wedge}\right)-\text { посылка } \\
& \mathrm{k}^{\prime}+3 . \quad A \vee(C \vee \neg C)-\supset_{\mathrm{n}}: \mathrm{k}^{\prime}, \mathrm{k}^{\prime}+2 \\
& \mathrm{k}^{\prime}+4 . \neg A-\supset_{\mathrm{n}}: \mathrm{k}^{\prime}+1, \mathrm{k}^{\prime}+2 \\
& \mathrm{k}^{\prime}+5 . \quad A-\text { посылка } \\
& \mathrm{k}^{\prime}+6 . \quad A \wedge \neg A-\wedge_{\mathrm{B}}: \mathrm{k}^{\prime}+4, \mathrm{k}^{\prime}+5 \\
& \mathrm{k}^{\prime}+7 .(A \wedge \neg A) \supset(C \vee \neg C)-\text { HPContPComp-аксиома } \\
& \mathrm{k}^{\prime}+8 . C \vee \neg C-\supset_{\mathrm{n}}: \mathrm{k}^{\prime}+6, \mathrm{k}^{\prime}+7 \\
& \mathrm{k}^{\prime}+9 . \quad A \supset(C \vee \neg C)-\text { теорема дедукции: } \mathrm{k}^{\prime}+8 \\
& \mathrm{k}^{\prime}+10 . \quad(C \vee \neg C) \supset(C \vee \neg C)-\text { HРContPComp-аксиома } \\
& \text { k' }+11 . \quad(A \supset(C \vee \neg C)) \supset(((C \vee \neg C) \supset(C \vee \neg C)) \supset((A \vee(C \vee \\
& \neg C)) \supset(C \vee \neg C))) \text { - HPContPComp-аксиома } \\
& \mathrm{k}^{\prime}+12 . C \vee \neg C-\supset_{\text {и }}: \mathrm{k}^{\prime}+3, \mathrm{k}^{\prime}+9, \mathrm{k}^{\prime}+10, \mathrm{k}^{\prime}+11 \text { (3 раза) } \\
& \mathrm{k}^{\prime}+13 .\left(\Gamma^{\wedge}\right) \supset(C \vee \neg C)-\text { теорема дедукции: } \mathrm{k}^{\prime}+12
\end{aligned}
$$

Q.E.D.

Теорема 7 следует из леммы 5 и $\Gamma \vdash_{\text {HPContPComp }} A \Leftrightarrow$ $\vdash_{H P C o n t P C o m p}\left(\Gamma^{\wedge}\right) \supset A$.

\section{Литература}

[1] Avron A. Natural 3-valued logics: characterization and proof theory // Journal of Symbolic Logic. 1991. Vol. 56. №1. P. 276-294.

[2] Бочаров В.А., Маркин В.И. Введение в логику: учебник. М.: ИД «Форум»: ИНФРА-М, 2008.

[3] Попов В.M. Секвенциальные формулировки паранепротиворечивых логических систем // Синтаксические и семантические исследования неэкстенсиональных логик (под ред. Смирнова В.А.). М.: Наука, 1989. С. 285-289.

[4] Попов B.M. Между Par и множеством всех формул // Материалы VI Международной конференции «Смирновские чтения» (отв. ред. Маркин В.И.). М.: Современные тетради, 2009. С. 93-95.

[5] Розоноэр Л.И. О выявлении противоречий в формальных теориях. I // Автоматика и телемеханика, №6. М.: Наука, 1983. С. 113-124. 
[6] Шангин B.O. Системы натурального вывода для логик Par, PCont, PComp и PContPComp // Материалы Научной конференции «Логика, методология, науковедение: актуальные проблемы и перспективы». Ростов-на-Дону, 2010.

[7] Шангин B.О. Системы натурального вывода для паранепротиворечивых логик, родственных логике, индуцированной исчислением V1 А. Арруда // Материалы Международной конференции «„Воображаемая логика“ Н.А. Васильева и современные неклассические логики». Казань, 2010. С. 106-108. 